\title{
Pseudo-cryptanalysis of the Original Blue Midnight Wish
}

\author{
Søren S. Thomsen* \\ DTU Mathematics, Technical University of Denmark \\ crypto@znoren.dk
}

\begin{abstract}
The hash function Blue Midnight Wish (BMW) is a candidate in the SHA-3 competition organized by the U.S. National Institute of Standards and Technology (NIST). BMW was selected for the second round of the competition, but the algorithm was tweaked in a number of ways. In this paper we describe cryptanalysis on the original version of BMW, as submitted to the SHA-3 competition in October 2008.

The attacks described are (near-)collision, preimage and second preimage attacks on the BMW compression function. These attacks can also be described as pseudo-attacks on the full hash function, i.e., as attacks in which the adversary is allowed to choose the initial value of the hash function. The complexities of the attacks are about $2^{14}$ for the nearcollision attack, about $2^{3 n / 8+1}$ for the pseudo-collision attack, and about $2^{3 n / 4+1}$ for the pseudo-(second) preimage attack, where $n$ is the output length of the hash function. Memory requirements are negligible. Moreover, the attacks are not (or only moderately) affected by the choice of security parameter for BMW.
\end{abstract}

Keywords: hash function cryptanalysis, SHA-3 competition, Blue Midnight Wish, pseudo-attacks.

\section{Introduction}

On October 31, 2008, the "SHA-3 competition", organized by the National Institute of Standards and Technology (NIST), was launched [1]. 64 algorithms were submitted, and 51 of these were accepted for the first round of the competition. On July 24, 2009, 14 candidates were chosen by NIST to advance to the second round of the competition.

One of the candidates that made it to the second round is called Blue Midnight Wish [2, or BMW for short. BMW was tweaked for the second round of the competition. Throughout this paper, unless explicitly stated otherwise, we always refer to the original version of the hash function, i.e., the version submitted to the competition before the October 31, 2008 deadline.

In this paper we describe some weaknesses in BMW. We show how to easily find near-collisions in the compression function of BMW. By near-collisions we

\footnotetext{
* The author is supported by a grant from the Villum Kann Rasmussen Foundation.
} 
mean a pair of inputs to the compression function for which the outputs only differ in a few (pre-specified) bit positions.

We also show how to find collisions, preimages, and second preimages in the compression function, faster than what is possible for an ideal compression function. This can be done by controlling 128, respectively 256 bits of the output of the compression function of BMW-256, respectively BMW-512. By controlling we mean that the adversary can give these bits any value he wishes with negligible effort. The complexity of these attacks corresponds to the complexity for a 192-bit, respectively 384-bit ideal hash function in the case of BMW-256, respectively BMW-512. Hence, for instance, pseudo-collisions can be found in BMW-512 in time about $2^{192}$, which is to be compared to the expected $2^{256}$ for an ideal 512-bit hash function. Memory requirements of all attacks are negligible.

We point out that the attacks described in this paper do not seem to apply to the tweaked version of Blue Midnight Wish. In Section 5, we briefly describe the tweaks and make some preliminary comments on these.

\section{A Description of Blue Midnight Wish}

Blue Midnight Wish is in fact a collection of four hash functions returning digests of four different sizes: 224 bits, 256 bits, 384 bits, and 512 bits. The two shortest digests are computed in the same way, except in the final step, which is a truncation. Likewise for the two longest digests. The word size, denoted by $w$, for the short variants is 32 bits, and for the long variants is 64 bits. Apart from the word size, all four variants are very similar. A little-endian byte ordering is assumed.

Blue Midnight Wish applies only four different types of operations: additions modulo $2^{w}$, exclusive-ors (XORs), and bitwise shifts and rotations. In the following, all additions of words in the description of Blue Midnight Wish are to be taken modulo $2^{w}$.

Blue Midnight Wish maintains a state of 16 words during the processing of a message; only in the end, the 16 words are truncated down to 6,7 , or 8 , depending on the digest size (truncation is done by throwing away the first 10,9 , or 8 words, respectively). Message blocks are also 16 words in length, and Blue Midnight Wish operates with a compression function mapping $2 \times 16$ words to 16 words. The compression function is iterated in a standard fashion [34]. Hence, the message $m$ of bitlength $\mu=|m|$ must be padded to a length that is a multiple of $16 \mathrm{w}$ bits, which is done by first appending a ' 1 '-bit, then appending $z=-\mu-65 \bmod 16 w^{\prime} 0$ '-bits (this part of the padding will be called " $10 \ldots$..." padding in the following), and finally appending a 64-bit representation of the message length $\mu$ (length padding). We now turn to a description of the Blue Midnight Wish compression function.

\subsection{The Blue Midnight Wish Compression Function}

The Blue Midnight Wish compression function takes two 16-word inputs and returns a single 16-word output. It applies three different sub-functions, which 
are called $P, f_{1}$, and $f_{2} . P$ is an efficiently invertible permutation $11 . f_{1}$ is a socalled multi-permutation taking two inputs, meaning that by fixing one of the inputs, the function is a permutation on the other input. Finally, $f_{2}$ compresses three inputs of 16 words to a single 16-word output, which is also the output of the compression function.

The two 16-word inputs to the compression function will be called $H$ and $M$, $H$ being the chaining variable, and $M$ being the message block. Referring to a single word in one of the inputs will be denoted by $H_{i}$ or $M_{i}$, meaning word number $i$, where counting starts from 0 . Hence, (e.g.) $M=M_{0}\left\|M_{1}\right\| \cdots \| M_{15}$.

The input to the permutation $P$ is $H \oplus M$. The output of $P$ is denoted $Y=Y_{0}\left\|Y_{1}\right\| \cdots \| Y_{15}$. The inputs to $f_{1}$ are $Y$ and $M$. The output of $f_{1}$ is denoted $Z=Z_{0}\left\|Z_{1}\right\| \cdots \| Z_{15}$. The inputs to $f_{2}$ are $Y, Z$, and $M$, and the output, which is also the output of the compression function, is denoted $H^{*}$. See also Fig. 1 .

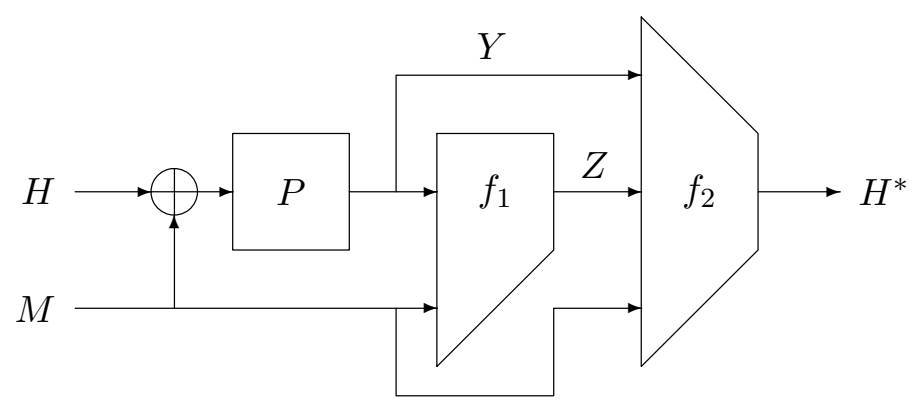

Fig. 1. The Blue Midnight Wish compression function

Given $Y$ and $M$, a matching $H$ can easily be computed as $P^{-1}(Y) \oplus M$, since $P$ is efficiently invertible. Moreover, $H$ is not used as input to any other subfunction than $P$. Hence, in attacks on the compression function, the details of $P$ are irrelevant, and therefore we do not describe these in this paper; see instead [2]. We now describe the two other components of the compression function.

A Description of $\boldsymbol{f}_{\mathbf{1}}$. As mentioned, the inputs to $f_{1}$ are $M$ and $Y$, and the output is denoted $Z$. Let $Q=Y \| Z$ be a 32 -word vector, and note that when $f_{1}$ is called, $Q$ contains 16 already computed words, and 16 "null" words. Then, $f_{1}$ can be described as a shift register, that computes one word of $Q$ at the time as a function of the previous 16 words of $Q$.

There are two variants of the step function that computes each new word of $Q$ : a simple step function, and a more complex one. Since 16 words are computed in $f_{1}$, there are always 16 rounds, but the number of complex and simple rounds

${ }^{1}$ In the Blue Midnight Wish specification, a mapping $f_{0}:\{0,1\}^{16 w} \times\{0,1\}^{16 w} \rightarrow$ $\{0,1\}^{16 w}$ is defined. Since $f_{0}$ is a permutation on the XOR of its two inputs, we choose to focus on this permutation and denote it $P$, i.e., $f_{0}(H, M)=P(H \oplus M)$. 
depends on a tunable security parameter. By default, there are first two complex rounds and then 14 simple rounds, and we shall generally assume that this is the distribution of complex and simple rounds. However, all our attacks apply to BMW using any value of the security parameter (in the case of the near-collision attack, some modifications are required).

Both complex and simple rounds use a number of invertible sub-functions $s_{0}, \ldots, s_{5}$ and $r_{1}, \ldots, r_{7}$, whose descriptions are postponed to Appendix $\mathrm{A}$, Both types of rounds also use the same "message schedule": consider $M$ to be a 16 element column vector in $\mathbb{Z}_{2}$, and define the matrix $\mathbf{B}$ as the circulant matrix whose first row contains the 16 elements

$$
[1,0,0,1,0,0,0,0,0,0,-1,0,0,0,0,0] .
$$

Row $i+1$ of $\mathbf{B}$ is row $i$ cyclically shifted one position to the right. Note that $\mathbf{B}$ is invertible for both word sizes; the inverses can be found in Appendix B. Define $W=\mathbf{B} \cdot M \bmod 2^{w}$, with $W_{i}$ referring to the $i$ th word of $W$. We note that this means that $W_{i}=M_{i}+M_{i+3}-M_{i+10} \bmod 2^{w}$, where the indices are to be reduced modulo 16 . In round $i$ of $f_{1}, W_{i}$ is involved in the computation of $Z_{i}=Q_{i+16}$.

Furthermore, 16 constants $K_{i}$ are defined as $\left\lfloor 2^{w} / 3\right\rfloor \cdot i$. We can now describe a complex round as

$$
\begin{aligned}
Q_{i+16} \leftarrow & s_{1}\left(Q_{i}\right)+s_{2}\left(Q_{i+1}\right)+s_{3}\left(Q_{i+2}\right)+s_{0}\left(Q_{i+3}\right) \\
& +s_{1}\left(Q_{i+4}\right)+s_{2}\left(Q_{i+5}\right)+s_{3}\left(Q_{i+6}\right)+s_{0}\left(Q_{i+7}\right) \\
& +s_{1}\left(Q_{i+8}\right)+s_{2}\left(Q_{i+9}\right)+s_{3}\left(Q_{i+10}\right)+s_{0}\left(Q_{i+11}\right) \\
& +s_{1}\left(Q_{i+12}\right)+s_{2}\left(Q_{i+13}\right)+s_{3}\left(Q_{i+14}\right)+s_{0}\left(Q_{i+15}\right) \\
& +W_{i}+K_{i},
\end{aligned}
$$

for increasing $i$; for the default choice of the tunable security parameter, $i$ increases from 0 to 1 . A simple round, covering the range of remaining values of $i$ up to and including 15 , is described as

$$
\begin{aligned}
Q_{i+16} \leftarrow & Q_{i}+r_{1}\left(Q_{i+1}\right)+Q_{i+2}+r_{2}\left(Q_{i+3}\right) \\
& +Q_{i+4}+r_{3}\left(Q_{i+5}\right)+Q_{i+6}+r_{4}\left(Q_{i+7}\right) \\
& +Q_{i+8}+r_{5}\left(Q_{i+9}\right)+Q_{i+10}+r_{6}\left(Q_{i+11}\right) \\
& +Q_{i+12}+r_{7}\left(Q_{i+13}\right)+s_{5}\left(Q_{i+14}\right)+s_{4}\left(Q_{i+15}\right) \\
& +W_{i}+K_{i} .
\end{aligned}
$$

Note that given $M$ and 16 consecutive words of $Q$, the remaining 16 words of $Q$ can be computed; in particular, given $M$ and $Z, Y$ can be computed. Likewise, given $Y$ and $Z$ (i.e., all of $Q$ ), $M$ can be computed via $W$ as $M=$ $\mathbf{B}^{-1} \cdot W \bmod 2^{w}$.

A Description of $\boldsymbol{f}_{\mathbf{2}}$. The sub-function $f_{2}$ takes as input $M, Y$, and $Z$. Let

$$
\begin{aligned}
& X_{L}=Z_{0} \oplus Z_{1} \oplus \cdots \oplus Z_{7} \quad \text { and } \\
& X_{H}=Z_{0} \oplus Z_{1} \oplus \cdots \oplus Z_{15} .
\end{aligned}
$$


The output words $H_{0}^{*}, \ldots, H_{15}^{*}$ are computed as follows.

$$
\begin{aligned}
& H_{0}^{*}=\left(X_{H}^{\ll 5} \oplus Z_{0}^{\gg 5} \oplus M_{0}\right)+\left(X_{L} \oplus Z_{8} \oplus Y_{0}\right) \\
& H_{1}^{*}=\left(X_{H}^{\gg 7} \oplus Z_{1}^{\ll 8} \oplus M_{1}\right)+\left(X_{L} \oplus Z_{9} \oplus Y_{1}\right) \\
& H_{2}^{*}=\left(X_{H}^{\gg 5} \oplus Z_{2}^{\ll 5} \oplus M_{2}\right)+\left(X_{L} \oplus Z_{10} \oplus Y_{2}\right) \\
& H_{3}^{*}=\left(X_{H}^{\gg} \oplus Z_{3}^{\ll 5} \oplus M_{3}\right)+\left(X_{L} \oplus Z_{11} \oplus Y_{3}\right) \\
& H_{4}^{*}=\left(X_{H}^{3} \oplus Z_{4} \oplus M_{4}\right)+\left(X_{L} \oplus Z_{12} \oplus Y_{4}\right) \\
& H_{5}^{*}=\left(X_{H}^{\ll 6} \oplus Z_{5}^{\gg 6} \oplus M_{5}\right)+\left(X_{L} \oplus Z_{13} \oplus Y_{5}\right) \\
& H_{6}^{*}=\left(X_{H}^{\gg 4} \oplus Z_{6} \ll 6 \oplus M_{6}\right)+\left(X_{L} \oplus Z_{14} \oplus Y_{6}\right) \\
& H_{7}^{*}=\left(X_{H}^{\gg 11} \oplus Z_{7}^{\ll 2} \oplus M_{7}\right)+\left(X_{L} \oplus Z_{15} \oplus Y_{7}\right) \\
& H_{8}^{*}=\left(H_{4}^{*}\right) \lll 9+\left(X_{H} \oplus Z_{8} \oplus M_{8}\right)+\left(X_{L}^{\ll 8} \oplus Z_{7} \oplus Y_{8}\right) \\
& H_{9}^{*}=\left(H_{5}^{*}\right) \lll 10+\left(X_{H} \oplus Z_{9} \oplus M_{9}\right)+\left(X_{L}^{\gg 6} \oplus Z_{0} \oplus Y_{9}\right) \\
& H_{10}^{*}=\left(H_{6}^{*}\right) \lll 11+\left(X_{H} \oplus Z_{10} \oplus M_{10}\right)+\left(X_{L}^{\ll 6} \oplus Z_{1} \oplus Y_{10}\right) \\
& H_{11}^{*}=\left(H_{7}^{*}\right)^{\ll 12}+\left(X_{H} \oplus Z_{11} \oplus M_{11}\right)+\left(X_{L}^{\ll 4} \oplus Z_{2} \oplus Y_{11}\right) \\
& H_{12}^{*}=\left(H_{0}^{*}\right)^{\ll 13}+\left(X_{H} \oplus Z_{12} \oplus M_{12}\right)+\left(X_{L}^{\gg 3} \oplus Z_{3} \oplus Y_{12}\right) \\
& H_{13}^{*}=\left(H_{1}^{*}\right)^{\ll 14}+\left(X_{H} \oplus Z_{13} \oplus M_{13}\right)+\left(X_{L}^{\gg 4} \oplus Z_{4} \oplus Y_{13}\right) \\
& H_{14}^{*}=\left(H_{2}^{*}\right)^{\ll 15}+\left(X_{H} \oplus Z_{14} \oplus M_{14}\right)+\left(X_{L}^{\gg 7} \oplus Z_{5} \oplus Y_{14}\right) \\
& H_{15}^{*}=\left(H_{3}^{*}\right)^{\ll 16}+\left(X_{H} \oplus Z_{15} \oplus M_{15}\right)+\left(X_{L}^{\gg 2} \oplus Z_{6} \oplus Y_{15}\right) .
\end{aligned}
$$

Here, $x^{\ll s}$ respectively $x^{\gg s}$ means $x$ shifted left, respectively right by $s$ bit positions. Similarly, $x^{\lll s}$ means $x$ rotated left by $s$ bit positions.

\section{Near-Collisions in the Compression Function}

Attacks on the compression function of Blue Midnight Wish are not affected by the permutation $P$, since this permutation can be inverted, and thereby the chaining input can be computed.

One may also observe that by choosing the same (XOR) differences in $H$ and $M$, there is no input difference in $P$, and therefore also no output difference. By ensuring that only the last few words of the expanded message $W$ contain a difference, we see that no difference is involved in a large part of $f_{1}$. Combined with the fact that diffusion is not very effective in $f_{2}$, this observation leads to near-collisions in the compression function.

Hence, a strategy to find the best (lowest weight) near-collision in the compression function is to search for difference patterns of the last few words of $W$, such that these differences do not spread too much in the last few rounds of $f_{1}$ and in $f_{2}$. Note that the inverse message schedule must be applied to $W$ in order to be able to compute $f_{2}$, and this message schedule will cause some 
diffusion of differences in $W$; however, differences in the most significant bits (MSBs) will remain in the MSB positions after the inverse message schedule is applied. Therefore, an obvious choice is to search for difference patterns in $W$ that only affect the MSBs of words of $W$.

\subsection{An Example}

In the case of both BMW-256 and BMW-512, the search mentioned showed that a good difference pattern in $W$ has differences in the MSBs of $W_{13}, W_{14}$, and $W_{15}$ only. The inverse message schedule causes differences in the MSBs of $M_{0}$, $M_{1}, M_{3}, M_{4}, M_{7}, M_{9}$, and $M_{13}$. Hence, there are 7 bit differences in $M$, which are introduced in the function $f_{2}$.

A difference in $W_{13}$ is propagated directly to $Z_{13}$ in the 13 th round of $f_{1}$. Hence, $Z_{13}$ obtains the difference $100 \ldots 0$ (in binary). In round 14 , the function $s_{4}$ is applied to $Z_{13}$ yielding the difference $1100 \ldots 0$ (see Appendix $\mathrm{A}$ ), and a difference in the MSB of $W_{14}$ is also introduced. The end result is the difference $0100 \ldots 0$ in $Z_{14}$ with probability $1 / 2$. Finally, in round 15 , the function $s_{5}$ is applied to $Z_{13}$ yielding the difference $10100 \ldots 0$, the function $s_{4}$ is applied to $Z_{14}$ yielding the difference $01100 \ldots 0$, and a difference in the MSB of $W_{15}$ is also introduced. Optimally, these differences result in the difference $1100 \ldots 0$ in $Z_{15}$, since then $\Delta Z_{13} \oplus \Delta Z_{14} \oplus \Delta Z_{15}=0$ ( $\Delta Z_{i}$ meaning the difference on $Z_{i}$ ), which means that in $f_{2}$, the variables $X_{L}$ and $X_{H}$ will contain no difference. The total probability of this characteristic is about $2^{-3}$. See Table 1 .

Table 1. The desired binary differences in the last three words of $Z$

\begin{tabular}{cc}
\hline Word & Desired XOR difference (binary) \\
\hline$Z_{13}$ & $100 \ldots 0$ \\
$Z_{14}$ & $010 \ldots 0$ \\
$Z_{15}$ & $110 \ldots 0$ \\
\hline
\end{tabular}

In $f_{2}$, as mentioned, the desired bit differences in $Z$ yield no difference in $X_{L}$ and $X_{H}$. Hence, in the output words $H_{0}^{*}, H_{1}^{*}, H_{3}^{*}, H_{4}^{*}$, there are only differences in the MSBs, and these come from the message $M$. There is no difference in $H_{2}^{*}$. In $H_{5}^{*}$, the MSB difference in $Z_{13}$ is inherited, and there are no other differences. In $H_{6}^{*}$, the difference $0100 \ldots 0$ in $Z_{14}$ is inherited and with probability $1 / 2$ does not propagate. In $H_{7}^{*}$, the MSB difference in $M_{7}$ cancels the MSB difference in $Z_{15}$, and the resulting difference is $0100 \ldots 0$, which does not propagate with probability $1 / 2$.

One may investigate in a similar way the effects on the words $H_{8}^{*}, \ldots, H_{15}^{*}$. This shows that as few as 17 bit differences remain in the best case, and this has a total probability of around $2^{-14}$. See Table 2. Note that in a pseudo-attack, only the last 6,7 , or 8 words are part of the output. 
Table 2. Output differences in the near-collision attack on the BMW compression function. Applies to all variants.

\begin{tabular}{cc}
\hline Word & XOR difference (binary) \\
\hline$H_{0}^{*}$ & $100 \ldots 00000000000000000$ \\
$H_{1}^{*}$ & $100 \ldots 00000000000000000$ \\
$H_{2}^{*}$ & $000 \ldots 00000000000000000$ \\
$H_{3}^{*}$ & $100 \ldots 00000000000000000$ \\
$H_{4}^{*}$ & $100 \ldots 00000000000000000$ \\
$H_{5}^{*}$ & $100 \ldots 00000000000000000$ \\
$H_{6}^{*}$ & $010 \ldots 00000000000000000$ \\
$H_{7}^{*}$ & $010 \ldots 00000000000000000$ \\
$H_{8}^{*}$ & $000 \ldots 00000000100000000$ \\
$H_{9}^{*}$ & $100 \ldots 00000001000000000$ \\
$H_{10}^{*}$ & $000 \ldots 00000001000000000$ \\
$H_{11}^{*}$ & $000 \ldots 00000010000000000$ \\
$H_{12}^{*}$ & $000 \ldots 000010000000000000$ \\
$H_{13}^{*}$ & $000 \ldots 00010000000000000$ \\
$H_{14}^{*}$ & $010 \ldots 00000000000000000$ \\
$H_{15}^{*}$ & $010 \ldots 010000000000000000$ \\
\hline
\end{tabular}

\subsection{Other Difference Patterns}

We note that the difference in $Z$ may be slightly different, and still give the same results as those described. For instance, the difference patterns of $Z_{14}$ and $Z_{15}$ may be swapped.

Moreover, there are in fact slightly better message difference patterns than the one described above. As an example, a difference in the MSB of $W_{13}$ and in the second-most significant bit of $W_{15}$ yields - with a high probability - a near-collision in all but 14 bits of the compression function output. However, the corresponding message difference in $M$ has a higher Hamming weight, and specifically there are differences in the words $M_{14}$ and $M_{15}$, which (in a pseudoattack on the hash function) are reserved for padding. We did not find simple difference patterns with differences only in the last few words of $W$ that lead to full collisions with high probability.

With a value of the security parameter above 13 , the above characteristic has a low (if not zero) probability. However, even with a value of 16 , a high probability characteristic exists producing near-collisions of total Hamming weight as low as 24 for the 16 output words of the compression function (see [5]). 


\subsection{A Pseudo-near-collision in BMW-256}

In the attack described above, there are no differences in $M_{14}$ and $M_{15}$, which in BMW-256 are the words containing length padding. This means we can extend the near-collision attack on the compression function to a pseudo-near-collision attack on the BMW-256 hash function. Moreover, one of the colliding messages may start from the correct initial value of BMW-256; the other initial value will be different from the correct one in the same 7 bit positions as those which contain differences in $M$.

As an example, the bit sequence of length 447 bits starting with the three bytes $\mathrm{f} 3 \mathrm{8b} 01$ and ending with (423) ' 0 '-bits follows the characteristic described above (with chaining input equal to the BMW-256 initial value). Further details can be found in the full version of this paper [6].

\section{Pseudo-attacks}

A second observation on the BMW compression function leads to improved pseudo-collision, -preimage, and -second preimage attacks: if $Z_{i}=0$ for all $i$, $0 \leq i<16$, then we get the following greatly simplified description of $f_{2}$.

$$
\begin{aligned}
H_{0}^{*} & =M_{0}+Y_{0} \\
H_{1}^{*} & =M_{1}+Y_{1} \\
H_{2}^{*} & =M_{2}+Y_{2} \\
H_{3}^{*} & =M_{3}+Y_{3} \\
H_{4}^{*} & =M_{4}+Y_{4} \\
H_{5}^{*} & =M_{5}+Y_{5} \\
H_{6}^{*} & =M_{6}+Y_{6} \\
H_{7}^{*} & =M_{7}+Y_{7} \\
H_{8}^{*} & =\left(M_{4}+Y_{4}\right)^{\lll 9}+M_{8}+Y_{8} \\
H_{9}^{*} & =\left(M_{5}+Y_{5}\right)^{\lll 10}+M_{9}+Y_{9} \\
H_{10}^{*} & =\left(M_{6}+Y_{6}\right)^{\ll 11}+M_{10}+Y_{10} \\
H_{11}^{*} & =\left(M_{7}+Y_{7}\right)^{\ll 12}+M_{11}+Y_{11} \\
H_{12}^{*} & =\left(M_{0}+Y_{0}\right)^{\lll 13}+M_{12}+Y_{12} \\
H_{13}^{*} & =\left(M_{1}+Y_{1}\right)^{\lll 14}+M_{13}+Y_{13} \\
H_{14}^{*} & =\left(M_{2}+Y_{2}\right)^{\ll 15}+M_{14}+Y_{14} \\
H_{15}^{*} & =\left(M_{3}+Y_{3}\right)^{\lll 16}+M_{15}+Y_{15} .
\end{aligned}
$$

\subsection{Controlling Output Words - A First Example}

Some output words of the compression function can be controlled by an attacker after fixing $Z=0$. The idea is to fix some words of $M$ and some words of $Y$ in such a way that a number of output words obtain an arbitrary value chosen by the attacker, and such that $f_{1}$ can be computed backwards, i.e., one may 
compute $Y_{15}$ from $Z$, then $Y_{14}$, etc. Words of $M$ can be fixed directly, since they are part of the input to the compression function. Words of $Y$ can be fixed indirectly via words of $W$, which, as mentioned, depend on $M$. There is enough freedom to fix some words of $M$ and some words of $W$ at the same time. More details follow. Note that this attack is independent of the value of the security parameter, since both simple and complex rounds are invertible.

Consider as an example the "new" definition of $H_{11}^{*}$ when $Z=0$ :

$$
H_{11}^{*}=\left(M_{7}+Y_{7}\right)^{\lll 12}+M_{11}+Y_{11}
$$

By fixing $M_{7}, Y_{7}, M_{11}$, and $Y_{11}$, one has effectively controlled $H_{11}^{*}$. Message words are part of the input to the compression function. Words of $Y$ can be controlled to some extent via words of $W$; after having fixed $Z$, we are able to compute words of $Y$ in the backward direction, i.e., we compute $Y_{15}$ first, then $Y_{14}$ etc., all the way down to $Y_{0}$. Alternatively, we can compute the value of $W_{i}$ needed to get some desired value of $Y_{i}$, for any $i$ such that $Y_{j}$ is already computed for all $j>i$. Thereby, we indirectly control $Y_{i}$.

As a simple example for BMW-256, assume we want $H_{11}^{*}$ to obtain the value $\alpha$. To do this, we may choose (e.g.) $M_{7}=Y_{7}=Y_{11}=0$ and $M_{11}=\alpha$. We obtain $Y_{7}=Y_{11}=0$ by controlling $W_{7}$ and $W_{11}$. Note that once $Y$ and $M$ are fixed, we compute $H$ as described in Section 2.1. How to control words of $M$ and words of $W$ at the same time is now described.

Sticking to the example, assume we want to be able to control $M_{7}, M_{11}, W_{7}$, and $W_{11}$. Compute $W=\mathbf{B} \cdot M$ with (initially) all words of $M$ as free parameters. As an example, one gets $W_{15}=M_{2}-M_{9}+M_{15}$. Now, make $W_{15}$ free by replacing everywhere $M_{15}$ by $W_{15}-M_{2}+M_{9}$. Now $W_{15}$ is freed, but $M_{15}$ is no longer free.

Since $W_{14}=M_{1}-M_{8}+M_{14}$, we can make $W_{14}$ free by replacing everywhere $M_{14}$ by $W_{14}-M_{1}+M_{8}$. We may continue like this, freeing all $W_{i}$ down to $i=7$ (incl.), without making $M_{7}$ or $M_{11}$ dependent. Since $M_{13}, M_{14}$, and $M_{15}$ contain padding, we might want to keep these three words of $M$ free as well. This way, one obtains (e.g.)

$$
\begin{aligned}
& W_{0}=-M_{1}+M_{3}+2 M_{7}-M_{13}-W_{7}+W_{13} \\
& W_{1}=2 M_{1}-M_{7}-M_{11}+M_{13}+W_{7}-W_{10} \\
& W_{2}=2 M_{1}-M_{3}+3 M_{11}+3 M_{14}+M_{15}-2 W_{8}-W_{9}-W_{11}-2 W_{14}-W_{15} \\
& W_{3}=-M_{1}+2 M_{3}-M_{11}-M_{13}-M_{14}+W_{8}+W_{9}-W_{12}+W_{14}+W_{15} \\
& W_{4}=M_{1}+M_{13}-M_{14}+W_{7}-W_{10} \\
& W_{5}=M_{1}+M_{11}+2 M_{14}-M_{15}-W_{11}-W_{14} \\
& W_{6}=M_{3}-M_{7}+M_{13}+M_{15}+W_{9}-W_{12}-W_{13} .
\end{aligned}
$$

All words appearing on the right hand sides are free, and all other words are dependent.

By computing the words $Y_{i}$ in the backward direction, or choosing $Y_{i}$ and computing the required $W_{i}$ for $i$ from 15 down to 7, we can control all the words $Y_{15}, Y_{14}, \ldots, Y_{7}$. In particular, we can make sure that $Y_{7}=Y_{11}=0$. Since $M_{7}$ 
and $M_{11}$ are free, we can also choose these two message words as we want; in particular, we can choose $M_{7}=0$ and $M_{11}=\alpha$, so that we obtain $H_{11}^{*}=\alpha$. Since we indirectly also control $H_{7}^{*}$, we can obtain $H_{7}^{*}=\beta$ for any $\beta$ of our choice via a proper choice of, say, $M_{7}$.

Note that in order to compute $Y_{1}$ and $Y_{0}, s_{1}$ must be inverted (see Section 2.1). This is slightly more complicated in practice than computing $s_{1}$ in the forward direction, but it can also be done efficiently (with some additional memory requirements) by pre-computing and storing all inverses.

The reason for choosing to control $H_{11}^{*}$ is that $Y_{7}$ is involved in its computation. This means we have to make only a few words of $W$ free $\left(W_{15}\right.$ down to $W_{7}$ ), and there is still a large amount of freedom in the choice of a number of words of $M$. This will be useful in extensions of the attack.

\subsection{Controlling Additional Output Words}

There are many degrees of freedom left. These can be used to control additional output words. For instance, we may control $H_{6}^{*}$ and $H_{10}^{*}$ via $M_{6}, W_{6}, M_{10}$, and $W_{10}$. We again keep $M_{14}$ and $M_{15}$ free as above, but $M_{13}$ is not free. We shall obtain correct "10..." padding in $M_{13}$ probabilistically; the probability is about $1 / 2$ if we assume only a single bit of " $10 \ldots$. " padding (hence, the message length is $512-65=447 \mathrm{bits}$ ). We set $M_{6}=M_{7}=M_{10}=M_{11}=0$ (for the sake of simplicity), and now we want to free all $W_{i}$ for $i$ from 15 down to 6 , since we need to be able to control $Y_{6}$. Using the same method as in the previous examples, we obtain

$$
\begin{aligned}
& W_{0}=2 M_{14}+M_{15}-W_{6}-2 W_{7}-2 W_{8}-W_{9}+W_{12}-2 W_{14}-2 W_{15} \\
& W_{1}=-M_{14}-M_{15}+W_{6}+W_{8}-W_{10}+W_{13}+W_{14}+W_{15} \\
& W_{2}=2 M_{14}+M_{15}-W_{7}-W_{8}-W_{11}-W_{12}-W_{14} \\
& W_{3}=2 M_{14}+M_{15}-W_{6}-2 W_{7}-2 W_{8}-W_{9}+W_{12}-W_{13}-2 W_{14}-2 W_{15} \\
& W_{4}=-2 M_{14}-M_{15}+W_{6}+W_{7}+W_{8}-W_{10}+W_{13}+W_{14}+W_{15} \\
& W_{5}=2 M_{14}-M_{15}-W_{7}-W_{11}-W_{14} .
\end{aligned}
$$

We now control the four output words $H_{6}^{*}, H_{7}^{*}, H_{10}^{*}$, and $H_{11}^{*}$ via $W_{6}, W_{7}, W_{10}$, and $W_{11}$. The time complexity of this attack is about 2 , since we need correct "10..." padding in $M_{13}$, but we have no (direct) control over this message word.

\subsection{Other Variants of BMW}

The same technique as described above for BMW-256 can be applied to BMW512. In fact, for BMW-512, length padding takes up only one message word, and therefore we have enough freedom to ensure correct "10..." padding with probability 1.

Obviously, the attacks also apply to BMW-224 and BMW-384, since these differ from BMW-256 and BMW-512 (respectively) only in the initial value and the final truncation. 


\subsection{Applications}

After truncation, two out of eight (or out of six or seven in the case of BMW-384 and BMW-224, respectively) output words can be given any value chosen by the attacker. This control can be used to carry out pseudo-attacks, i.e., attacks in which the attacker is free to choose the initial value of the hash function. Example pseudo-attacks are pseudo-collision, pseudo-preimage, and pseudo-second preimage attacks. The time complexities of these attacks on BMW correspond to brute force attacks on $3 / 4$ of the output bits (or $2 / 3$ or $5 / 7$ in the case of BMW-384 and BMW-224, respectively). Hence, the time complexity is reduced compared to an ideal hash function. Table 3 summarizes the attack complexities for the three types of attack on the four variants of Blue Midnight Wish. Memory requirements are negligible.

As mentioned, pseudo-attacks are attacks in which the attacker is free to choose the initial value of the hash function. In the case of pseudo-collision and pseudo-second preimage attacks, the two colliding messages will generally assume two different initial values.

Table 3. Pseudo-attack complexities on the four Blue Midnight Wish variants (in brackets, birthday/brute force complexities)

\begin{tabular}{cccc}
\hline Variant & \multicolumn{2}{c}{ Pseudo-collision } & Pseudo-(second) preimage \\
\hline BMW-224 & $2^{81}$ & $\left(2^{112}\right)$ & $2^{161}\left(2^{224}\right)$ \\
BMW-256 & $2^{97}\left(2^{128}\right)$ & $2^{193}\left(2^{256}\right)$ \\
BMW-384 & $2^{128}\left(2^{192}\right)$ & $2^{256}\left(2^{384}\right)$ \\
BMW-512 & $2^{192}\left(2^{256}\right)$ & $2^{384}\left(2^{512}\right)$ \\
\hline
\end{tabular}

\subsection{Available Degrees of Freedom}

Clearly, in these attacks we do not have to choose $Z$ to be all-zero, we can choose it to be anything we want. Also, we have lots of freedom in the choice of $M_{6}, M_{7}$, $M_{10}, M_{11}, H_{6}^{*}$, and $H_{7}^{*}$ to get the desired values of $H_{10}^{*}$ and $H_{11}^{*}$. The choices we made in the examples above were only to simplify expressions. The available degrees of freedom may be useful in extensions of the attacks; however, so far we did not succeed in doing this.

\subsection{Examples}

For examples demonstrating the attack, see the full version of this paper [6].

\section{The Tweaked Blue Midnight Wish}

For the second round of the SHA-3 competition, Blue Midnight Wish was tweaked in three ways [7: 
1. The function $f_{0}(H, M)$, which in the original BMW was defined as $P(H \oplus M)$, is now defined as $P(H \oplus M)+H^{\lll w} \bmod 2^{w}$.

2. The message expansion in $f_{1}$ is changed. The terms $M_{i}+M_{i+3}-M_{i+10}+$ $K_{i}$, as they appeared in the original BMW, are replaced by $\left(M_{i} \lll 1+i+\right.$ $\left.M_{i+3} \lll 1+(i+3 \bmod 16)-M_{i+10} \lll 1+(i+10 \bmod 16)+K_{i} \bmod 2^{w}\right) \oplus H_{i+7}$.

3 . In the tweaked version, after the processing of the message, the compression function is invoked again, using a constant value for $H$ and using the intermediate hash of the message as $M$.

The first tweak means that it is still easy to compute $M$ given $H$ and $Y$, but now it appears to be hard to compute $H$ given $M$ and $Y$ (as needed in the attacks described above). The second tweak seems to imply that one needs to choose $H$ before $f_{1}$ can be computed, in contrast with the original case, where $H$ did not have to be chosen until the complete attack on the compression function had been carried out. The last tweak makes it harder to turn some compression function attacks into pseudo-attacks.

Some preliminary thoughts with respect to cryptanalysis of the tweaked BMW follow.

- Collisions in $f_{0}$ with constant $M$ may now exist, since it is no longer guaranteed that $f_{0}$ is a multi-permutation.

- Since each output word of $f_{0}$ depends on only six words of $H$, one can find an input $H$ (for arbitrary $M$ ) such that eight out of 16 output words of $f_{0}$ have any desired value. The complexity of this "attack" is 1 , and it allows the attacker to choose eight words of $H$ arbitrarily.

- $f_{1}$ is still a multi-permutation, i.e., given two out of three inputs and the output, the last input can be (efficiently) computed. It may be of particular interest that given any $M, Y, Z$, a matching $H$ in $f_{1}$ is easy to compute.

Aumasson [8] found a distinguisher for the Tweaked Blue Midnight Wish compression function requiring $2^{19}$ unknown input pairs with a fixed difference. The distinguisher detects a strong bias on the least significant bits of the output word $H_{0}^{*}$. Similarly, Guo and Thomsen [9] provided input differences to the tweaked BMW compression function such that with a limited amount of message modification, a single or a few output bits contain a difference with probability 0 or 1 (depending on the difference chosen). These distinguishers do not threaten the security of the hash function.

\section{Conclusion}

We have described a number of weaknesses in the original version of the Blue Midnight Wish hash function.

The weaknesses lead to attacks in which the adversary is allowed to choose the initial value of the hash function. It is by no means straightforward to extend the attacks to full-blown attacks using the given initial values of the BMW variants. Meet-in-the-middle attacks also do not seem possible since BMW uses an internal state that is at least twice as large as the output of the hash function. 
The attacks, as they are described in this paper, apparently do not apply to the tweaked version of BMW.

Acknowledgments. I would like to thank Guo Jian and my colleagues at DTU Mathematics for useful feedback and encouragement, and the anonymous reviewers for helpful comments.

\section{References}

1. National Institute of Standards and Technology: The SHA-3 competition website available, http://csrc.nist.gov/groups/ST/hash/sha-3/index.html (2009/08/26)

2. Gligoroski, D., Klíma, V., Knapskog, S.J., El-Hadedy, M., Amundsen, J., Mjølsnes, S.F.: Cryptographic Hash Function Blue Midnight Wish. SHA-3 Algorithm Submission (October 2008), http://csrc.nist.gov/groups/ST/hash/sha-3/Round1/documents/ Blue_Midnight_Wish.zip (2009/11/09)

3. Damgård, I.: A Design Principle for Hash Functions. In: Brassard, G. (ed.) CRYPTO 1989. LNCS, vol. 435, pp. 416-427. Springer, Heidelberg (1990)

4. Merkle, R.C.: One Way Hash Functions and DES. In: Brassard, G. (ed.) CRYPTO 1989. LNCS, vol. 435, pp. 428-446. Springer, Heidelberg (1990)

5. Thomsen, S.S.: A near-collision attack on the Blue Midnight Wish compression function. Manuscript (November 2008), http://www.mat.dtu.dk/people/S.Thomsen/bmw/nc-compress.pdf (2009/09/09)

6. Thomsen, S.S.: Pseudo-cryptanalysis of the Original Blue Midnight Wish. Cryptology ePrint Archive, Report 2009/478 (2009), http://eprint.iacr.org/

7. Gligoroski, D., Klíma, V.: A Document describing all modifications made on the Blue Midnight Wish cryptographic hash function before entering the Second Round of SHA-3 hash competition (September 2009), http://people.item.ntnu.no/ danilog/Hash/BMW-SecondRound/ Supporting_Documentation/Round2Mods.pdf (2009/11/09)

8. Aumasson, J.P.: Practical distinguisher for the compression function of Blue Midnight Wish. Manuscript, http://131002.net/data/papers/Aum10.pdf (2010/03/10)

9. Guo, J., Thomsen, S.S.: Distinguishers for the Compression Function of Blue Midnight Wish with Probability 1. Manuscript (March 2010), http://www.mat.dtu.dk/people/S.Thomsen/bmw/bmw-distinguishers.pdf (2010/03/31) 


\section{A Sub-functions Used in $f_{1}$}

The sub-functions $s_{i}, 0 \leq i \leq 4$, and $r_{i}, 1 \leq i \leq 7$, used in $f_{1}$ are defined as follows.

\begin{tabular}{|c|c|}
\hline BMW-224 and BMW-256 & BMW-384 and BMW-512 \\
\hline$s_{0}(x)=x^{\gg 1} \oplus x^{\ll 3} \oplus x^{\lll 4} \oplus x^{\lll 19}$ & $s_{0}(x)=x^{\gg 1} \oplus x^{\ll 3} \oplus x^{\lll 4} \oplus x^{\ll 37}$ \\
\hline$s_{1}(x)=x^{\gg 1} \oplus x^{\ll 2} \oplus x^{\lll 8} \oplus x^{\lll 23}$ & $s_{1}(x)=x^{\gg 1} \oplus x^{\ll 2} \oplus x^{\lll 13} \oplus x^{\lll 43}$ \\
\hline$s_{2}(x)=x^{\gg 2} \oplus x^{\ll 1} \oplus x^{\lll 12} \oplus x^{\lll 25}$ & $s_{2}(x)=x^{\gg 2} \oplus x^{\ll 1} \oplus x^{\lll 19} \oplus x^{\lll 53}$ \\
\hline$s_{3}(x)=x^{\gg 2} \oplus x^{\ll 2} \oplus x^{\lll 15} \oplus x^{\lll 29}$ & $s_{3}(x)=x^{\gg 2} \oplus x^{\ll 2} \oplus x^{\lll 28} \oplus x^{\lll 59}$ \\
\hline$s_{4}(x)=x^{\gg 1} \oplus x$ & $s_{4}(x)=x^{\gg 1} \oplus x$ \\
\hline$s_{5}(x)=x^{\gg 2} \oplus x$ & $s_{5}(x)=x^{\gg 2} \oplus x$ \\
\hline$r_{1}(x)=x^{\lll 3}$ & $r_{1}(x)=x^{\lll 5}$ \\
\hline$r_{2}(x)=x^{\lll 7}$ & $r_{2}(x)=x^{\lll 11}$ \\
\hline$r_{3}(x)=x^{\lll 13}$ & $r_{3}(x)=x^{\lll 27}$ \\
\hline$r_{4}(x)=x^{\lll 16}$ & $r_{4}(x)=x^{\lll 32}$ \\
\hline$r_{5}(x)=x^{\lll 19}$ & $r_{5}(x)=x^{\lll 37}$ \\
\hline$r_{6}(x)=x^{\lll 23}$ & $r_{6}(x)=x^{\lll 43}$ \\
\hline$r_{7}(x)=x^{\lll 27}$ & $r_{7}(x)=x^{\lll 53}$ \\
\hline
\end{tabular}

\section{B Inverses of the Matrix B Used in $f_{1}$}

The matrix $\mathbf{B}$ is introduced in Section 2.1. This matrix is circulant, meaning that each row is equal to the row above rotated one position to the right. The inverses modulo $2^{32}$ and $2^{64}$ are also circulant. The first row of $\mathbf{B}^{-1} \bmod 2^{32}$ (in hexadecimal) is

[abababac, c6c6c6c7, bdbdbdbe, c0c0c0c1, 15151515, 4e4e4e4e, 90909090, cfcfcfd0, babababb, 6c6c6c6d, dbdbdbdc, 0c0c0c0c, 51515151, e4e4e4e5, 09090909, fcfcfcfd].

The first row of $\mathbf{B}^{-1} \bmod 2^{64}$ is

[abababababababac, c6c6c6c6c6c6c6c7, bdbdbdbdbdbdbdbe, c0c0c0c0c0c0c0c1, 1515151515151515, 4e4e4e4e4e4e4e4e, 9090909090909090, cfcfcfcfcfcfcfd0, babababababababb,

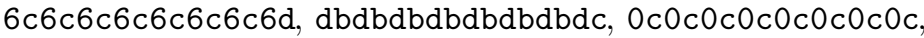
5151515151515151, e4e4e4e4e4e4e4e5, 0909090909090909 , $f c f c f c f c f c f c f c f d]$. 